\title{
Radial extracorporeal shockwave therapy on calcaneal spurs: a randomized controlled trial
}

\author{
Desy Kurniawati Tandiyo, ${ }^{1}$ Ratna Darjanti Haryadi, ${ }^{2}$ Ari Probandari, ${ }^{3}$ Didik Gunawan Tamtomo ${ }^{4}$
}

pISSN: 0853-1773 • elSSN: 2252-8083 https://doi.org/10.13181/mji.v28i4.3039 Med J Indones. 2019;28:316-21

Received: August 13, 2018

Accepted: August 15, 2019

\section{Authors' affiliations:}

${ }^{1}$ Doctoral Program in Medicine, Faculty of Medicine, Universitas Sebelas Maret, Surakarta, Indonesia, ${ }^{2}$ Department of Physical Medicine and Rehabilitation, Faculty of Medicine, Universitas Airlangga, Dr. Soetomo Hospital, Surabaya, Indonesia, ${ }^{3}$ Department of Public Health, Faculty of Medicine, Universitas Sebelas Maret, Surakarta, Indonesia, ${ }^{4}$ Department of Anatomy, Faculty of Medicine, Universitas Sebelas Maret, Surakarta, Indonesia

Corresponding author:

Desy Kurniawati Tandiyo Faculty of Medicine, Universitas Sebelas Maret, Jalan Ir. Sutami No. 36A, Surakarta 57126, Indonesia Tel/Fax: +62-71-663377

E-mail: tandiyo.desy@yahoo.co.id

\begin{abstract}
BACKGROUND The effectiveness of extracorporeal shockwave therapy (ESWT) in handling pain and calcaneal spurs is still controversial, whereas research on the effectiveness of ESWT in the reactive ossification process of calcaneal spurs, involving osteoprotegerin (OPG) and the receptor activator of nuclear factor-kappa B (RANK), does not exist. This study was aimed to assess the effect of ESWT on pain, the length of the spur, plasma OPG and RANK level on the calcaneal spur.
\end{abstract}

METHODS This study was a randomized controlled trial in patients with calcaneal spurs. ESWT was administered at the pain point by applying 2,000 shocks, at an intensity of 2 bars, given six times with a 7-10-day interval. The visual analog scale (VAS) data, plasma OPG and RANK level were analyzed using Mann-Whitney $U$ test, whereas spur size was analyzed using chi-square test.

RESULTS One month after therapy at rest $(p<0.001)$ and when walking after getting up $(p=0.020)$, the VAS was lower than that in the control group. The plasma OPG level was lower than control group shortly after therapy $(p<0.001)$. The plasma RANK level was higher than control group shortly after therapy $(p<0.001)$. ESWT did not affect the reduction of spur measurement $(p=0.382)$.

CONCLUSIONS ESWT reduced pain, decreased plasma OPG level, and increased plasma RANK level. Although ESWT did not have any effect on spur reduction, it affected plasma OPG and RANK level that play a role in the activity of osteoblasts and osteoclasts.

KEYWORDS calcaneal spur, extracorporeal shockwave therapy
Heel spurs were first reported in 1900 by Plettner, a German doctor, who gave them the term Kalkaneussporn (calcaneal spurs)., ${ }^{1,2}$ A spur is an extra bone. It is formed at the attachment site of ligaments or tendons to bone, which grows in the direction of the pull of the ligaments or tendons. ${ }^{3-6}$ Spur prevalence in the UK population is $38 \%,{ }^{6}$ whereas in the Indian population the incidence of calcaneal spurs was $26.5 \%$ of the 200 calcanei studied. ${ }^{2}$ According to Cailliet, ${ }^{3}$ recurrent traction of the insertion of the fascia plantaris on the calcaneus leads to the process of inflammation and reactive ossification. The pathological process involves the production of small tears produced from the stretching of the plantar tendon fibers on the periosteum that are attached to the heel resulting in subperiosteal inflammation. At the same time, the damage is replaced by fibrous tissue and calcium deposits forming a spur. ${ }^{3}$ Rogers 
et $\mathrm{al}^{4}$ suggest that the formation of this bone is a response of the musculoskeletal system to stress and injury according to Wolff's law of bone remodeling.

Bone remodeling involves the synthesis of bone matrix by osteoblast cells and its resorption by osteoclast cells.7,8 An imbalance between osteoblasts and osteoclasts has immunopathological implications associated with decreased and increased bone mineral density. The balance between three molecular factors composed of osteoprotegerin (OPG), receptor activator of nuclear factor-kappa $B$ ligand (RANKL), and RANK, maintains physiologic bone remodeling. ${ }^{9}$

Some options of conservative therapy, such as modality therapy, are aimed at reducing pain, but not reducing spurs.5,10,11 Usually, to reduce or eliminate a spur, surgery is performed. ${ }^{12}$ Alternative therapy to reduce pain and spurs, namely extracorporeal shockwave therapy (ESWT), is now available. ${ }^{13}$

The effectiveness of ESWT in the treatment of heel pain and calcaneal spurs is controversial, whereas research on the effectiveness of ESWT in the reactive ossification process of calcaneal spurs involving the OPG and RANK is still not available. This study was aimed to assess the effect of ESWT on pain, the length of the spur, plasma OPG, and the levels of RANK on calcaneal spurs.

\section{METHODS}

This study used the experimental randomized controlled trial method in patients with calcaneal spurs at the Medical Rehabilitation Installation, Dr. Moewardi Hospital, Surakarta, Indonesia. This study was approved by the Health Research Ethics Committee, Faculty of Medicine, Universitas Sebelas Maret (No: 119/II/HERC/2017), and written informed consent was obtained from every patient.

\section{Study population}

The subjects of the study were patients who were clinically and radiologically diagnosed with calcaneal spurs and met the study criteria. The inclusion criteria were patients with heel pain, adults (age $\geq 20$ years old), foot $x$-rays showing images of spurs, a 1-week modalityfree therapy period (before visual analog scale [VAS] measurement), an analgesic-free period for 48 hours (before VAS measurement), and were cooperative and willing to participate in this research program by signing the consent form. Participants with an active infection in the treated area, an open wound on the treated area, a fracture in the treated area, a history of malignancy, pregnancy, and an impaired sensory ability in the treated area were excluded. Drop out criteria were not adhering to the therapy schedule at least once, having

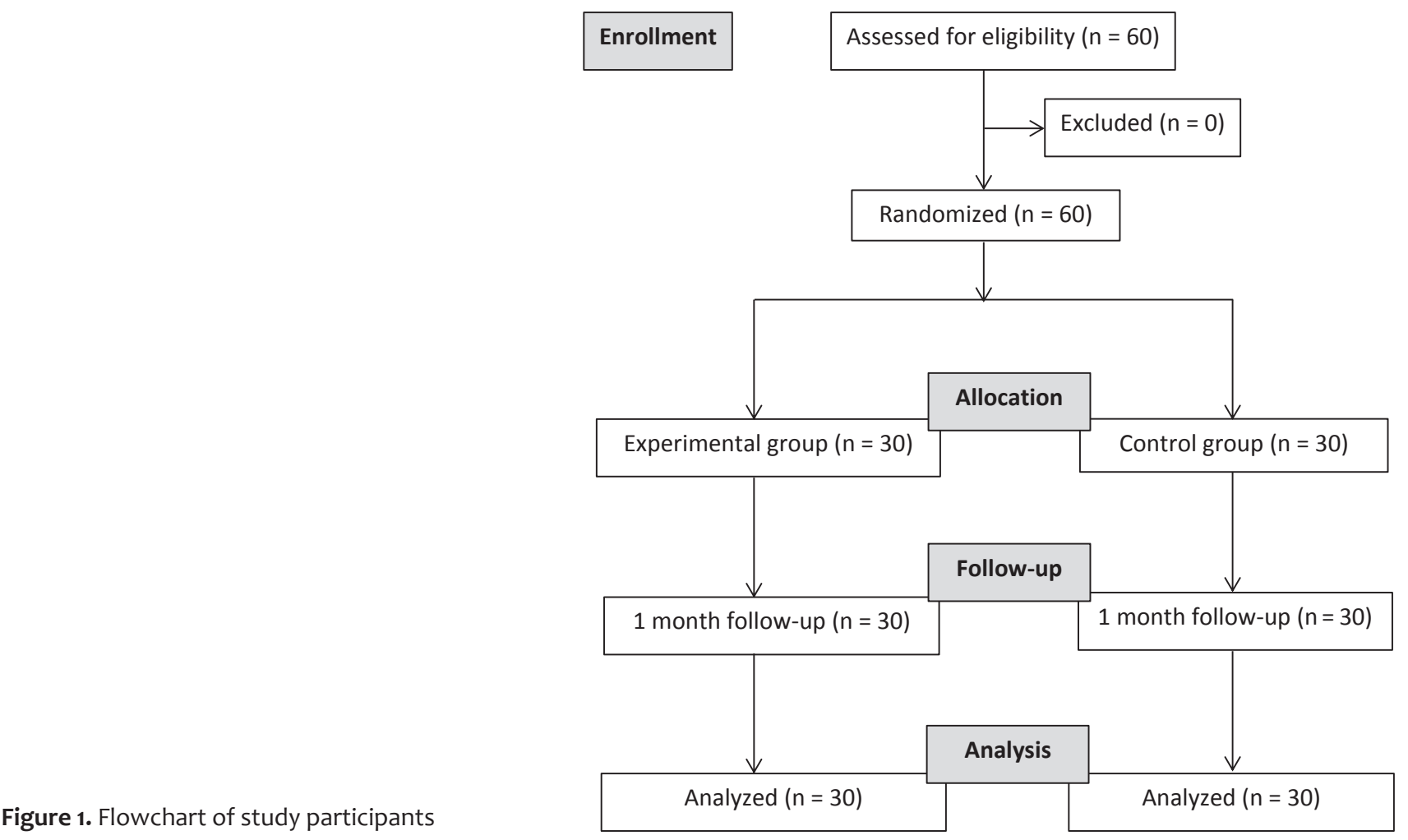

Figure 1. Flowchart of study participants 
other diseases that contraindicate therapy during their treatment, and having complications that might arise due to the therapy. The subjects were allocated into two groups: 1) the treatment group receiving ultrasound diathermy (USD) and ESWT, and 2) the control group receiving USD alone (Figure 1). All groups received acetaminophen $500 \mathrm{mg}$ three times daily starting on the first day of treatment and continuing for 5 days ( 15 tablets).

\section{Interventions}

\section{Extracorporeal shockwave therapy (ESWT) procedure}

In this study, 2,000 shocks were given at the point of pain using a BTL-6000 SWT (BTL Industries Ltd, Bulgaria) with the SWT radial technique, at an intensity of 2 bars, six times with intervals of 7-10 days between sessions.

\section{Ultrasound diathermy (USD) procedure}

USD is sound wave therapy given at the point of pain using an Sonopuls 490 (Enraf-Nonius, Netherlands) had a non-thermal effect, in continuous mode, at a frequency of $0.1 \mathrm{~W} / \mathrm{cm}^{2}$, for $10 \mathrm{~min}$, and performed six times with intervals of 3-5 days.

\section{Outcomes}

\section{Pain level}

Pain level was measured by a visual analog scale (VAS). VAS scores range from $0=$ no pain to $10=$ maximal pain. VAS scores were measured with VAS graphical sheet ${ }^{14}$ by researcher at rest, while walking after getting up, and after activity. VAS measurements were performed before, shortly after the six session, and one month after therapy.

\section{Spur length}

According to the length of radiological features, spurs were grouped into spur reduction $<1 \mathrm{~mm}$ and $\geq 1 \mathrm{~mm}$. Radiological features are taken with the heel in the lateral position. Foot x-ray and radiological measurements by radiologist using tools in the software (X-ray Toshiba KXO-32S, CR-1824) were performed before the first therapy, and one month after the last therapy.

\section{Osteoprotegerin (OPG) and receptor activator of nuclear factor-kappa B (RANK) plasma level \\ OPG and RANK plasma level was measured with an enzyme-linked immunosorbent assay (ELISA), in duplo.}

The blood was taken from the cubital vein before therapy and shortly after therapy, and measurement with OPG Human ELISA Kit (Thermo Fisher Scientific ${ }^{\mathrm{TM}}$, Maryland) and RANK Human ELISA Kit (Thermo Fisher Scientific ${ }^{\mathrm{TM}}$, Maryland).

\section{Sample size}

The OpenEpi program (http://www.openepi.com) was used to identify a suitable size for the intervention. The input parameters were $7 \%$ of unexposed with outcomes; $40 \%$ of exposed with outcomes; an a-error of 0.05 ; and with a power of $80 \%$. The percentages of unexposed with outcomes and exposed with outcomes were calculated by a previous study. ${ }^{13}$ The results showed that the treatment group and the control group required 25 patients each. Hence, a total of 60 patients were enrolled, allowing for possible dropouts.

\section{Statistical analysis}

VAS data, OPG, and RANK plasma level were analyzed using the Mann-Whitney $U$ test, whereas spur measurements were analyzed using the chisquare test. A $p<0.05$ was considered statistically significant.

\section{RESULTS}

General characteristics of study participants are presented in Table 1. Different VAS scores were obtained from measurements after one month of therapy at rest in the treatment group (median = $1)$, and in the control group (median $=3)(p<0.001)$ (Figure 2a); and when walking after getting up in the treatment group (median 4$)$, and in the control group (median $=4.5)(p=0.020)$ (Figure $2 b)$. There was similar VAS score between the control and treatment group prior before and after therapy at rest, when walking after getting up, and after an activity (Figure 2c).

ESWT could not reduce spur length $(>1 \mathrm{~mm})$. The plasma level of OPG shortly after therapy was lower in the treatment group than the control group $(p<0.001)$. Similarly, the average difference in the plasma level of OPG was lower in the treatment group than the control group ( $p=0.007)$. Besides, the difference in the plasma level of RANK was higher in the treatment group than the control group $(p<$ 0.001). Furthermore, the average difference in the plasma level of RANK in the treatment group was 
Table 1. Characteristics of the subjects in the study and spur measurement reduction on the radiological image, OPG, and RANK plasma level differences between the treatment and control group

\begin{tabular}{|c|c|c|c|}
\hline Variables & Control, n (\%) (N = 30) & Treatment, $\mathrm{n}(\%)(\mathrm{N}=30)$ & $p$ \\
\hline Male sex & $3(10)$ & $3(10)$ & \\
\hline \multicolumn{4}{|l|}{ Occupancy } \\
\hline Active & $21(70)$ & $19(63)$ & \\
\hline Housewife & $5(17)$ & $6(20)$ & \\
\hline Retired & $4(13)$ & $5(17)$ & \\
\hline \multicolumn{4}{|l|}{ Calcaneus } \\
\hline Left & $16(53)$ & $11(37)$ & \\
\hline Right & $14(47)$ & $19(63)$ & \\
\hline Age (years), mean (SD) & $50.87(10.19)$ & $48.77(9.92)$ & \\
\hline Weight $(\mathrm{kg})$, mean (SD) & $63.1(8.88)$ & $66.1(12.28)$ & \\
\hline Height $(\mathrm{cm})$, mean (SD) & $155.33(5.35)$ & $156.33(6.04)$ & \\
\hline \multicolumn{4}{|l|}{ Spur reduction } \\
\hline$<1 \mathrm{~mm}$ & 24 & 20 & $0.243^{*}$ \\
\hline$\geq 1 \mathrm{~mm}$ & 6 & 10 & \\
\hline \multicolumn{4}{|c|}{ OPG (pg/ml), median (min-max) } \\
\hline Before therapy & $171.84(84.67-340.56)$ & $162.93(60.86-281.77)$ & $0.198^{+}$ \\
\hline Shortly after therapy & $216.47(112.45-425.01)$ & $153.43(99.25-309.71)$ & $<0.001^{+}$ \\
\hline$\Delta$ & $35.64(-149.79-202.17)$ & $-7.95(-89.86-68.07)$ & $0.007^{+}$ \\
\hline \multicolumn{4}{|c|}{ RANK (ng/ml), median (min-max) } \\
\hline Before therapy & $0.57(0.21-7.46)$ & $0.56(0.35-1.41)$ & $0.734^{+}$ \\
\hline Shortly after therapy & $0.4(0.23-7.22)$ & $0.58(0.40-2.49)$ & $<0.001^{+}$ \\
\hline$\Delta$ & $-0.18(-0.55-2.49)$ & $0.03(-0.65-1.08)$ & $0.032^{+}$ \\
\hline
\end{tabular}

OPG=osteoprotegerin; RANK=receptor activator of nuclear factor-kappa B; SD=standard deviation *Chi-square test; ${ }^{\dagger}$ Mann-Whitney $U$ test

higher than the control group $(p=0.032)$ (Table 1).

\section{DISCUSSION}

The effect of ESWT in pain measured with VAS was found one month after treatment in every condition (at rest, when walking after getting up, and after an activity). Several studies described the effect of ESWT on pain level..$^{11,13,15-19}$ Cosentino et $\mathrm{al}^{13}$ reported giving ESWT therapy six times, once a week, with 1,200 shocks at a frequency of 120 shocks/sec, and at various energy densities from 0.03 to $0.4 \mathrm{~mJ} /$ $\mathrm{mm}^{2}$, resulted in significant pain reduction at the end of therapy, one month, and three months later at rest, while walking after getting up, and after daily activities. Gollwitzer et al ${ }^{19}$ reported that giving 250 patients ESWT at a frequency of $0.25 \mathrm{~mJ} / \mathrm{mm}^{2}$, with 2,000 shocks, for three sessions with weekly intervals proved ESWT effectiveness in handling heel pain. The results showed a significant reduction of pain in the
ESWT group (69.2\%) compared with the control group (34.5\%) in 12 weeks after the last therapy session. In contrast, Buchbinder et al ${ }^{16}$ reported that ESWT 1,000 $\mathrm{mJ} / \mathrm{mm}^{2}$ given once a week within three weeks, did not provide any evidence to support the benefits of ESWT regarding pain, function, and quality of life in patients with heel pain. In this study, the effect of ESWT in the VAS were measured 1 month following treatment at rest and while walking after getting up in the treatment and control groups was likely because the effect of ESWT could be seen in a long-term. Biological reactions and recovery effect of ESWT could not occur immediately; and improvements are obtained in the medium- or long-term. ${ }^{20}$

In this study, there was no significant ESWT effect on spur reduction. In contrast, Cosentino et $\mathrm{al}^{13}$ reported that giving ESWT therapy six times, once per week, by applying 1200 shocks at a frequency of 120 shocks/sec, and at various energy densities from 0.03 to $0.4 \mathrm{~mJ} / \mathrm{mm}^{2}$, resulted in significant spur reduction 

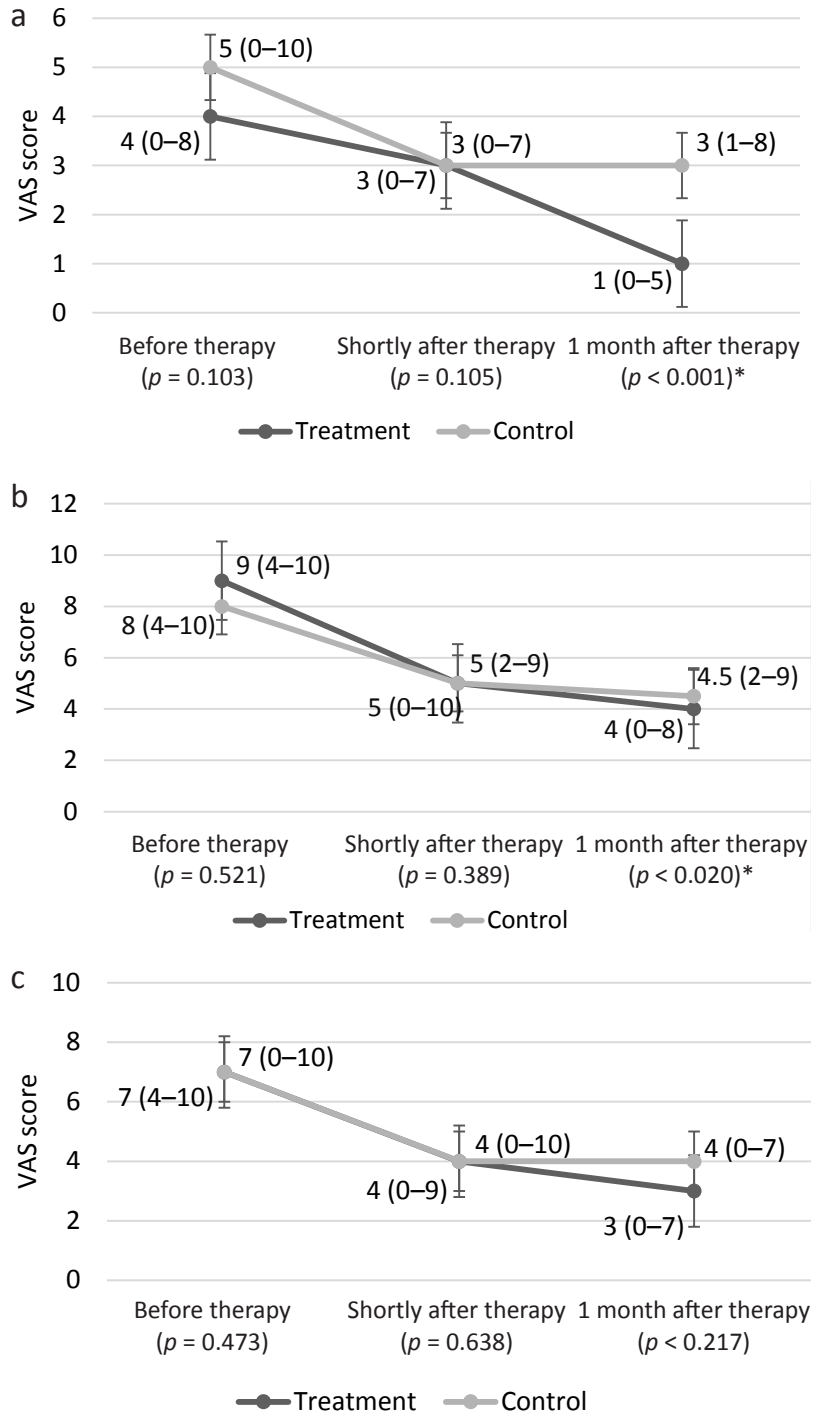

Figure 2. Differences in the visual analog scale (VAS) between the control group and the treatment group before therapy, shortly after therapy, and one month after therapy. (a) Median VAS (min-max) at rest. (b) Median VAS (min-max) when walking after getting up. (c) Median VAS (min-max) after an activity. $p$-values were calculated between treatment and control group using Mann-Whitney $U$ test. *significant if $p<0.05$

of the radiological features measured 1 month after therapy in the treatment group.

In the study, there was a reduction in spurs $\geq 1$ $\mathrm{mm}$ in 10 subjects compared with the control group in which there was a reduction of spurs $>1 \mathrm{~mm}$ in 6 subjects. This result is more likely because of the ESWT effect on the focus of therapy that led to fragmentation and cavitation and resulted in the disorganization and disintegration of deposits, ${ }^{21}$ and the mechanism of calcification material loss in ESWT is caused by improved metabolism because of increased blood circulation and vascularization, ${ }^{22}$ whereas the treatment and control groups received USD therapy, which also has a cavitation effect and improved metabolism. ${ }^{25}$

In this study, plasma OPG level was lower than control group and reduced shortly after therapy. It means there was a decrease of osteoblasts formation after therapy. Plasma RANK level was higher than in the control group and increased shortly after therapy. It means there was an increase of osteoclast formation after therapy. An imbalance between osteoblasts and osteoclasts has immunopathological implications associated with decreased and increased bone mineral density. The balance between three molecular factors consisting of OPG, RANKL, and RANK maintains physiologic bone remodeling. ${ }^{9}$ The plasma level of OPG decreased in the treatment group, and the plasma level of RANK increased in the treatment group before therapy and shortly after therapy. The imbalance between OPG and RANK was probably related to the size of spurs regarding radiological features. Although in this study, ESWT did not have any effect on the reduction of spurs on radiological images, it influenced plasma OPG and the levels of RANK that play a role in osteoblast and osteoclast activity.

Several studies have described the effects of ESWT on OPG, ${ }^{23,24}$ but there were no study on the effects of ESWT on RANK. Huang et a ${ }^{23}$ reported that ESWT has beneficial effects on osteoporotic fracture healing in rats. It may promote the expression of OPG and bone morphogenetic protein-2 (BMP-2) in the osteoporotic fracture area in rats. BMP-2 and OPG may act synergistically and lead to significantly enhanced bone formation and remodeling. Hence, there were no study on the effectiveness of ESWT in the process of reactive ossification on calcaneal spurs involving OPG and RANK.

This study has several limitations. First, it only measured the VAS before therapy, shortly after therapy, and one month after therapy because of other uncontrollable factors that affect the VAS in long-term follow-up, such as patient compliance and medication use, or other therapy modalities (especially in the control group), whereas the effect of ESWT occurs in the medium or long term. ${ }^{20}$ Second, in this study, the treatment and control groups received USD therapy, which also has a cavitation effect, ${ }^{25}$ whereas ESWT effect mechanisms on calcification seems probable because of the increased pressure on focus areas of therapy, which leads to fragmentation and cavitation, 
resulting in the disorganization and disintegration of deposits. ${ }^{21}$ Therefore, the effect of USD cavitation might affect the results of this study.

In this study, ESWT reduced pain (VAS). It did not have any effect on spur reduction, but it decreased plasma levels of OPG, and increased plasma levels of RANK. This suggests that ESWT may still have potential effect in spur reduction because OPG and RANK play important roles in osteoblast and osteoclast activity.

\section{Conflict of Interest}

The authors affirm no conflict of interest in this study.

\section{Acknowledgment}

The authors would like to thank Mr. Dono Indarto, M.D., Ph.D., Head of Biomedics Laboratory, Universitas Sebelas Maret, and Mrs. Rachmi Fauziah Rahayu, M.D., Sp. Rad. for their strong support and assistance, also for their invaluable ideas and time.

\section{Funding Sources}

None.

\section{REFERENCES}

1. Menz HB, Zammit GV, Landorf KB, Munteanu SE. Plantar calcaneal spurs in older people: longitudinal traction or vertical compression? J Foot Ankle Res. 2008;1(1):7.

2. Kullar JS, Randhawa GK, Kullar KK. A study of calcaneal enthesophytes (spurs) in Indian population. Int J Appl Basic Med Res. 2014;4(Suppl 1):S13-6.

3. Cailliet R. Soft tissue pain and disability. $2^{\text {nd }}$ ed. Philadelphia: F.A. Davis Co; 1988. p. 307-65.

4. Rogers J, Shepstone L, Dieppe P. Bone formers: osteophyte and enthesophyte formation are positively associated. Ann Rheum Dis. 1997;56(2):85-90.

5. Thomas JL, Christensen JC, Kravitz SR, Mendicino RW, Schuberth JM, Vanore JV, et al. The diagnosis and treatment of heel pain: a clinical practice guideline-revision 2010. J Foot Ankle Surg. 2010;49(3 Suppl):S1-19.

6. Toumi H, Davies R, Mazor M, Coursier R, Best TM, Jennane R, et al. Changes in prevalence of calcaneal spurs in men \& women: a random population from a trauma clinic. BMC Musculoskelet Disord. 2014;15:87.

7. Lacey DL, Timms E, Tan HL, Kelley MJ, Dunstan CR, Burgess T, et al. Osteoprotegerin ligand is a cytokine that regulates osteoclast differentiation and activation. Cell. 1998;93(2):165-76.

8. Bezerra MC, Carvalho JF, Prokopowitsch AS, Pereira RM. RANK, RANKL and osteoprotegerin in arthritic bone loss. Braz J Med Biol Res. 2005;38(2):161-70.

9. Kohli SS, Kohli VS. Role of RANKL-RANK/osteoprotegerin molecular complex in bone remodeling and its immunopathologic implications. Indian J Endocrinol Metab.
2011;15(3):175-81.

10. Stuber K, Kristmanson K. Conservative therapy for plantar fasciitis: a narrative review of randomized controlled trials. J Can Chiropr Assoc. 2006;50(2):118-33.

11. Gollwitzer H, Diehl P, von Korff A, Rahlfs VW, Gerdesmeyer L. Extracorporeal shock wave therapy for chronic painful heel syndrome: a prospective, double blind, randomized trial assessing the efficacy of a new electromagnetic shock wave device. J Foot Ankle Surg. 2007;46(5):348-57.

12. Gautham P, Nuhmani S, Kachanathu SJ. Plantar fasciitis: a review of literature. Saudi J Sports Med. 2014:14(2):69-73.

13. Cosentino R, Falsetti P, Manca S, De Stefano R, Frati E, Frediani $B$, et al. Efficacy of extracorporeal shock wave treatment in calcaneal enthesophytosis. Ann Rheum Dis. 2001;60(11):1064-7.

14. Kumar $P$, Tripathi L. Challenges in pain assessment: pain intensity scales. Indian J Pain. 2014;28:61-70.

15. Weil LS Jr, Roukis TS, Weil LS, Borelli AH. Extracorporeal shock wave therapy for the treatment of chronic plantar fasciitis: indications, protocol, intermediate results, and comparison of results to fasciotomy. J Foot Ankle Surg. 2002;41(3):166-72.

16. Buchbinder R, Ptasznik R, Gordon J, Buchanan J, Prabaharan $V$, Forbes A. Ultrasound-guided extracorporeal shock wave therapy for plantar fasciitis: a randomized controlled trial. JAMA. 2002;288(11):1364-72.

17. Thomson CE, Crawford F, Murray GD. The effectiveness of extra corporeal shock wave therapy for plantar heel pain: a systematic review and meta-analysis. BMC Musculoskelet Disord. 2005;6:19.

18. Malay DS, Pressman MM, Assili A, Kline JT, York S, Buren B, et al. Extracorporeal shockwave therapy versus placebo for the treatment of chronic proximal plantar fasciitis: results of a randomized, placebo-controlled, double-blinded, multicenter intervention trial. J Foot Ankle Surg. 2006;45(4);196-210.

19. Gollwitzer H, Saxena A, DiDomenico LA, Galli L, Bouché RT, Caminear DS, et al. Clinically relevant effectiveness of focused extracorporeal shock wave therapy in the treatment of chronic plantar fasciitis: a randomized, controlled multicenter study. J Bone Joint Surg Am. 2015;97(9):701-8.

20. Dreisilker U. Significance of ESWT in practice. In: Dreisilker U. Shock wave therapy in practice. Enthesiopathies. Heilbronn: Leveho Buchverlag Daniela Bamberg; 2010. p. 16-20.

21. Notarnicola A, Moretti B. The biological effects of extracorporeal shock wave therapy (eswt) on tendon tissue. Muscles Ligaments Tendons J. 2012;2(1):33-7.

22. Dreisilker U. History: An overview. In: Dreisilker U. Shock wave therapy in practice. Enthesiopathies. Heilbronn: Leveho Buchverlag Daniela Bamberg; 2010. p. 21-7.

23. Huang HM, Li XL, Tu SQ, Chen XF, Lu CC, Jiang LH. Effects of roughly focused extracorporeal shock waves therapy on the expressions of bone protein-2 and osteoprotegerin in osteoporotic fracture in rats. Chin Med J. 2016;129(21):2567-75.

24. Notarnicola A, Tamma R, Moretti L, Fiore A, Vicenti G, Zallone $A$, et al. Effects of radial shock wave therapy on osteoblast activities. Musculoskelet Surg. 2012;96(3):183-9.

25. Draper DO, Prentice WE. Therapeutic ultrasound. In: Prentice WE. Therapeutic modalities in rehabilitation. Fourth edition. New York: The McGraw-Hill Companies, Inc; 2011. p. 363-416. 